Revista de Psicología y Educación / Journal of Psychology and Education, 2019, 14(1), 16-26 (www.rpye.es)

\title{
Patrón de uso de internet y control parental de redes sociales como predictor de sexting en adolescentes: una perspectiva de género Víctor José Villanueva-Blasco ${ }^{*}$ y Sara Serrano-Bernal ${ }^{2}$
}

'Universidad Internacional de Valencia

${ }^{2}$ Universidad de Zaragoza

Resumen: El presente estudio analiza desde una perspectiva de género la relación entre el patrón de uso de internet, la medida de control parental aplicada a redes sociales y el sexting en una muestra de adolescentes. Es un estudio descriptivo-relacional de tipo transversal. Participaron 163 adolescentes entre 12 y 16 años. Los hallazgos muestran que una mayor frecuencia de conexión a internet puede ser un factor de riesgo para sexting y sextorsión, pero únicamente para mujeres. Por su parte, el control parental de redes sociales se relaciona con una menor frecuencia de conexión a internet tanto en hombres como en mujeres. Sin embargo, cuando se combinan control parental de redes sociales con medidas restrictivas de frecuencia de horas de conexión a internet y franja horaria nocturna de conexión, disminuye la probabilidad de exposición al sexting y la sextorsión, pero únicamente en mujeres. Se señala la necesidad de impulsar estrategias educativas y preventivas a nivel familiar y escolar que fomenten un uso adecuado de internet, y promuevan la autorregulación de su uso en adolescentes.

Palabras clave: Sexting, Control parental, Redes sociales, Adolescencia, Sextorsión.

\section{Pattern of internet use and parental monitoring of social networks as a predictor of sexting in adolescents: a gender perspective}

Abstract: This study analyze from a gender perspective the relationship between the pattern of internet use, the measure of parental monitoring applied to social networks and sexting in a sample of adolescents. It is a cross-sectional descriptive-relational study. There were 163 adolescents between 12 and 16 years old. Findings show that a higher frequency of internet connection can be a risk factor for sexting and sextorsión, but only for female. On the other hand, the parental monitoring of social networks is related to a lower frequency of internet connection in both, men and female. However, when parental control of social networks is combined with restrictive measures of frequency of hours internet connection and night time connection, the probability of exposure to sexting and sextorsión decreases, but only in female. Educational and preventive strategies are needed at the family and schools that encourage an adequate internet use, and promote self-regulation of its use in adolescents.

Keywords: Sexting, Parental monitoring, Social networks, Adolescence, Sextorsión.

Las Tecnologías de la Información y de la Comunicación (en adelante TIC) han cambiado la forma de comunicación de las personas así como la forma de relacionarse, llegando a formar parte de sus vidas (Lau y Yuen, 2016). En relación a los adolescentes, internet tiene un papel importante vinculado a procesos

Recibido: 30/05/2018 - Aceptado: 27/09/2018 - Avance online: 17/10/2018 *Correspondencia: Víctor José Villanueva Blasco.

Universidad Internacional de Valencia.

C.P: 44003, Teruel, España.

E-mail: vivillanueva@universidadviu.com educativos y de socialización, e influyendo en su sentido de identidad, normas morales, valores y principios (Gómez, Rial, Braña, Golpe y Varela, 2017). Internet les ofrece la posibilidad de comunicarse a través de las redes sociales y mensajes de texto (Moodley y Singh, 2016), crear blogs, vídeos, páginas web para el desarrollo de la propia competencia personal, mantener relaciones de amistad, así como actividades educativas y de promoción y prevención de la salud (Arab y Díaz, 2015). 
Diversos autores (De la Villa Moral y Suárez, 2016; Echeburúa, 2013), coinciden en afirmar que los adolescentes son los que con mayor frecuencia utilizan las TIC debido a su alta familiarización con ellas, por eso se trata de un grupo de riesgo ante el posible mal uso de internet ya que continuamente buscan nuevas experiencias a través de ésta. Según el informe EU Kids Online (Garmendia, Jiménez, Casado y Mascheroni, 2016), resulta complicado definir qué actividades de la red son beneficiosas y cuales son perjudiciales. En todo caso, la preocupación de los padres ha aumentado en los últimos tiempos debido al uso y actividades relacionadas con internet que tienen sus hijos (Fernández-Montalvo, Peñalva e Irazabal, 2015).

Internet permite crear nuevas relaciones virtuales tanto con amistades conocidas como con desconocidos, permitiendo el ocultamiento de la identidad personal gracias al anonimato, lo que lleva a un aumento de la probabilidad de desarrollar problemas en las relaciones interpersonales (Fernández-Montalvo et al., 2015). El estudio de Gómez et al. (2017) revela que contactar con desconocidos es el comportamiento más frecuente entre los adolescentes, y aumenta en aquellos usuarios que tienen menos control sobre el uso de internet. En el estudio de Garmendia et al. (2016), siete de cada diez jóvenes aceptan como amigos sólo a los que conocen (52\%) o a aquellos que conocen muy bien (17\%); en cambio, un $23 \%$ acepta a personas con las que tiene amistades en común y un $9 \%$ a todos los que les agregan.

En cuanto a la existencia de diferencias de género, diversos estudios encuentran que las chicas utilizan más las redes sociales mientras que los chicos pasan más tiempo con el juego online (Chang et al., 2015; Fernández-Montalvo et al., 2015). Sin embargo, Garmendia et al. (2016) hallaron que a pesar de que las chicas son más propensas a aceptar todo tipo de peticiones de amistad, también suelen ser las que más aceptan solo a aquellas personas que conocen o conocen muy bien.

Entre los riesgos más destacados en las redes sociales se encuentran el ciberbullying, sexting, grooming o el fomento de ideas o mensajes inadecuados (Echeburúa, 2013; Gómez et al. 2017) pudiendo llegar a ser perjudiciales para los adolescentes, quienes se encuentran en un periodo de desarrollo personal en el que destaca y se prioriza la experimentación de nuevas emociones y situaciones.

Centrando el presente trabajo en el sexting como uno de los riesgos de internet menos estudiados, este ha sido definido por Arab y Díaz (2015, p. 10) como "una práctica que consiste en compartir imágenes de tipo sexual, personal o de otros, por medio de teléfonos o de internet". Garmendia et al. (2016) encontraron que un $31 \%$ de los jóvenes ha recibido mensajes con contenido sexual, observando diferencias en cuanto al género, habiendo recibido este tipo de mensajes un $28 \%$ de las chicas frente al 35\% de los chicos. En este estudio se señala que el sexting, como la mayoría de las actividades relacionadas con internet, aumenta con la edad en el rango de edades del estudio (9-16 años), con una prevalencia del 19\% en menores de $11-12$ años, del $34 \%$ entre los 13 14 años y del $42 \%$ para los $15-16$ años. Estos mensajes con contenido sexual, en la mayoría de los casos en todas las edades, se recibían a través de los mensajes instantáneos (entre el $15 \%$ y $35 \%$ según la edad) y, en menor medida por las redes sociales (6\%). Como señalan estos mismos autores, el sexting se desarrolla en la mayor parte de las ocasiones dentro de la interacción entre pares contextualizado en la experimentación de la identidad sexual y como acción con connotaciones sexuales. Sin embargo, existe un riesgo de que las imágenes y textos sean publicados y viralizados sin permiso en espacios online, y fácilmente distribuidas a través de mensajería instantánea o redes sociales, derivándose con ello problemáticas tales como el ciberacoso o la sextorsión.

Ante la posibilidad de exposición a riesgos como el sexting, diversos autores señalan la importancia de la participación de la familia en la educación y prevención de los usos inadecuados de internet. Lau y Yuen (2016) destacan que los padres y madres deberían conocer cuáles son los riesgos de internet a los que pueden 
acceder sus hijos y así tomar las medidas más adecuadas para prevenir el mal uso. Como señalan Mascheroni, Murru, Aristodemou y Laouris (2013), la familia, a través de acciones educativas basadas en la regulación de los beneficios y riesgos de internet, promueven el empoderamiento y autorregulación en sus hijos. En definitiva, aquellos adolescentes que estén más instruidos y sean más competentes en el uso de las herramientas de internet, es más probable que sean conscientes de los riesgos que existen, evalúen las diferentes situaciones y riesgos, mostrando un uso con menor probabilidad de exposición a los riesgos de internet.

La definición de control parental (parental monitoring o parental mediation) varía según los estudios que se tomen de referencia, utilizándose asimismo en la literatura el término mediación parental. Ambos no son sinónimos, sino que se atiende a una distinta categorización de las estrategias reguladoras que puede ejercer la familia. Podemos concretar que el control parental se corresponde con el modelo de mediación parental restrictiva, que se basa en el establecimiento de normas y límites que restringen el uso del medio, incluidas las restricciones relativas al tiempo y al contenido, con el fin de que los menores aprendan de forma progresiva a navegar de manera segura sin la compañía de un adulto (Garmendia et al., 2016). Entre las acciones de control parental podemos considerar el informarse acerca de los contenidos a los que acceden sus hijos, monitorizar sus actividades en internet y con quiénes tienen relación en redes sociales, limitar el tiempo de uso, o restricciones técnicas, entre otras.

Aunque en la actualidad el control parental no es una práctica muy común, es considerado un factor de protección sobre los riesgos de internet, sobre todo en relación al tiempo de conexión (Leung y Lee, 2011), siendo ésta la acción a la que se limita dicho control más que a los contenidos a los que acceden o con quien mantienen relaciones (Leung y Lee, 2011; Sureda, Comas y Morey, 2010). Duerager y Livingstone (2012) señalan que el control parental es lo que conlleva menor exposición a riesgos, sin limitar las oportunidades que tienen los menores de beneficiarse del entorno online.

Asimismo, si los adolescentes perciben un alto control parental, experimentarán lo que se ha denominado presencia parental, la cual facilita que internalicen las ideas y normas propuestas por sus padres y, progresivamente, alcanzar la autorregulación ante el uso de internet (Ding, Li, Zhou, Dong y Luo, 2017). Además, los adolescentes que señalan tener más control por parte de sus padres, sienten más involucración positiva por parte de estos en su vida (Khurana, Bleakley, Jordan, y Romer, 2015).

A pesar de que el control parental es más efectivo durante la adolescencia, los resultados muestran que tiene efecto protector tanto para los más jóvenes como para los más mayores, y para ambos sexos (Khurana et al., 2015). Sin embargo, Arnaiz, Cerezo, Giménez y Maquilónz (2016), señalan diferencias de género en el control parental, indicando que son las chicas quienes informan estar expuestas a un mayor control parental.

La literatura sobre el sexting dispone de escasos estudios abordados desde una perspectiva de género, por lo que en el presente estudio la incorporamos como objetivo principal en el análisis de la relación entre el patrón de uso de internet, la medida de control parental aplicada a redes sociales y el sexting en una muestra de adolescentes.

Asimismo, siendo el sexting una de las prácticas de riesgo en el uso de internet menos estudiadas, el presente estudio permitirá ahondar en el conocimiento de algunos de los factores de riesgo y protección asociados, orientando así hacia estrategias educativas y de prevención más efectivas que contemplen las diferencias de género existentes.

\section{MÉTODO}

\section{DISEÑO DEL ESTUDIO}

Se trata de un estudio descriptivo-relacional de tipo transversal, realizado en un contexto natural en centros educativos de enseñanza secundaria durante el curso académico 2017. 18, con alumnado de $1^{\circ}$ a $4^{\circ}$ curso de E.S.O. 


\section{PARTICIPANTES}

La muestra se compone de 163 alumnos de 12 a 16 años, cuya edad media es de 13,63 años (DT $=1,18)$, de un Instituto de Educación Secundaria (IES) de titularidad concertada, de la ciudad de Calatayud (España). La muestra se compone por 68 mujeres $(41,7 \%)$ y 95 hombres (58,3\%), y la distribución por cursos académicos es en $1^{\circ}$ ESO 35 alumnos $(21,5 \%)$, en $2^{\circ}$ ESO 50 alumnos $(30,7 \%)$, en $3^{\circ}$ ESO 36 alumnos $(22,1 \%)$, y en $4^{\circ} \mathrm{ESO} 42$ alumnos $(25,8 \%)$.

\section{INSTRUMENTOS DE MEDIDA}

Cuestionario sociodemográfico. Elaborado ad hoc, permite evaluar datos relativos al género y edad, así como información sobre el centro educativo, grupo y aula a la que pertenece.

Escala de frecuencia de horas de uso de internet y franja horaria de uso. Elaborada ad hoc, se compone de un ítem relativo a la frecuencia de horas de uso "En los últimos 30 días, ¿̇cuántas horas al día te has conectado para utilizar WhatsApp, Youtube, juegos online u otras aplicaciones?", cuyo formato posee seis opciones de respuesta: Menos de una (1); Entre una y dos (2); Entre dos y tres (3); Entre tres y cinco (4); Más de cinco (5); y Durante todo el día (6). Una mayor puntuación es un indicador de una mayor frecuencia de uso de internet. Se complementa con cuatro ítems relativos al uso de internet en diferentes franjas horarias: "En los últimos 30 días, Zen qué franja horaria te has conectado a internet?", solicitando que señalen en cada franja horaria "De 8 de la mañana a 2 de la tarde", "De 2 de la tarde a 9 de la noche", "De 9 de la noche a 12 de la noche" y "A partir de las 12 de la noche", ofreciendo tres opciones de respuesta: Nada (1); A veces (2); y Todos o casi todos los días (3). Una mayor puntuación es un indicador de una mayor frecuencia de conexión a internet en dicha franja horaria.

Escala de exposición a riesgos en las redes sociales. Elaborada ad hoc, se compone de
6 ítems relativos a situaciones de riesgo en internet y redes sociales. En las instrucciones se solicita a los participantes que señalen si les has sucedido alguno de los hechos que se señalan (ver Tabla 1) durante los últimos 30 días. La variable es de tipo cuantitativo con cinco opciones de respuesta: Nunca (1); A veces (1-5 veces al mes) (2); A menudo (2-3 veces por semana) (3); Todos o casi todos los días (4); y Todos los días varias veces (5). Una mayor puntuación es un indicador de una mayor frecuencia de exposición a situaciones de riesgo en las redes sociales.

Escala de control parental del uso de internet y el teléfono móvil. Elaborada ad hoc, se compone de 9 ítems relativos a las conductas de control de sus padres/ madres respecto a su uso de internet y teléfono móvil (por ejemplo, "Mis padres me controlan en qué redes sociales entro y con quién mantengo conversaciones a través de internet"). En las instrucciones se solicita a los participantes que señalen que afirmación se ajusta más a cada uno de ellos y el grado de acuerdo. La variable es de tipo cuantitativo con 5 opciones de respuesta: Totalmente en desacuerdo (1); En desacuerdo (2); No lo sé (3); De acuerdo (4); y Totalmente de acuerdo (5). Una mayor puntuación es un indicador de un mayor control parental.

\section{PROCEDIMIENTO}

La selección del centro fue no aleatoria. Primero se contactó con el IES participante para informar del estudio y solicitar a través de éste el consentimiento informado de los padres, madres o tutores legales. La administración de los instrumentos se realizó en el aula ordinaria en horario de tutoría, con respuesta individual por parte del alumnado. Se les informó a los participantes de la voluntariedad de su participación y de la importancia de la sinceridad en las respuestas, garantizándoles la confidencialidad de estas a través de una clave alfanumérica. La duración estimada para la administración de la batería de instrumentos es de 30 a 40 minutos. 


\section{ANÁLISIS DE DATOS}

Todos los análisis se realizaron con el paquete estadístico Statistical Package for the Social Sciences (SPSS) versión 21. En primer lugar, se realizó un análisis descriptivo de la muestra, calculando los índices de tendencia central (media y desviación estándar). Este primer análisis permite una caracterización de la muestra, así como las puntuaciones para cada una de las variables de estudio.

En segundo lugar, se realizó un análisis de correlaciones bivariadas entre la variable Sexting (evaluada a través del ítem "Alguien que he conocido en una red social me ha enviado contenidos de tipo sexual (fotos, vídeos) sin pedírselo", Solicitud de sexting, Horas de conexión, Franja horaria (de 21 a 24 horas y a partir de las 24 horas) y Control parental de las redes sociales y con quien mantienen relación online. El estadístico que se reporta es el valor de Pearson.

Finalmente, se realizó un análisis de la covarianza (ANCOVA) para determinar cuáles de las variables de estudio actuaban como predictoras del sexting. Se consideraron como variables dependientes Sexting y Solicitud de sexting. Para ello, se han tratado dichas variables como si fuesen cuantitativas atendiendo a que su valor establece una frecuencia de experimentación de sexting y solicitud de sexting en el intervalo temporal contemplado. Como variables independientes se contemplaron Frecuencia de conexión a internet, Franja horaria de conexión (conexión de 21 a 24 horas, y conexión a partir de las 24 horas) y Control parental de las redes sociales. Asimismo, los análisis se realizaron segregando el archivo en base a la variable género. El estadístico utilizado en las pruebas multivariante fue la Lambda de Wilks, aportando los valores de la prueba de Levene $(F)$, la significación (p) y el Eta parcial al cuadrado $\left(\eta^{2}\right)$.

\section{RESULTADOS}

Los resultados sobre la exposición a riesgos relacionados con el sexting en las redes sociales se presentan en la Tabla 1, mostrando las prevalencias tanto para la muestra total como en función del género.

Los resultados de frecuencia de conexión y franja horaria de conexión, se recogen en la Tabla 2. Destaca que el $25.8 \%$ de los menores se conectan con un máximo de dos horas, el $42.4 \%$ entre dos y cinco horas y el $31.9 \%$ lo hace más de cinco horas o a lo largo de todo el día.

En cuanto al análisis de correlaciones (Tabla 3), en la submuestra de chicos la frecuencia de conexión a internet correlacionó positivamente con la conexión de 21 a 24 horas $(r=.53$; $p<.001)$ y a partir de las 24 horas $(r=.56$; $p<.001)$, y negativamente con control parental

Prevalencia de exposición a riesgos en las redes sociales

\begin{tabular}{|c|c|c|c|}
\hline & $\begin{array}{l}\text { Hombres } \\
(n=95)\end{array}$ & $\begin{array}{l}\text { Mujeres } \\
(n=68)\end{array}$ & $\begin{array}{c}\text { Total } \\
(N=163)\end{array}$ \\
\hline & $f(\%)$ & $f(\%)$ & $f(\%)$ \\
\hline $\begin{array}{l}\text { Alguien que he conocido en una red social me ha enviado } \\
\text { contenidos de tipo sexual (fotos, videos...) sin pedírselo }\end{array}$ & $9(9.5)$ & $9(13.2)$ & $18(11)$ \\
\hline $\begin{array}{l}\text { Alguien que he conocido en una red social me ha pedido que le } \\
\text { envíe fotos o vídeos míos de tipo sexual }\end{array}$ & $6(6.3)$ & $9(13.2)$ & $15(9.2)$ \\
\hline $\begin{array}{l}\text { Alguien con quien había compartido fotos o vídeos míos de tipo } \\
\text { sexual me ha chantajeado para que le envíe más o los difundirá }\end{array}$ & $0(0)$ & $1(1.5)$ & $1(0.6)$ \\
\hline
\end{tabular}


Tabla 2

Prevalencia respecto a horas al día de conexión a internet y franja horaria

\begin{tabular}{|c|c|c|}
\hline \multicolumn{2}{|c|}{} & $\begin{array}{c}\text { Total } \\
(\mathrm{N}=163)\end{array}$ \\
\cline { 3 - 3 } & & $f(\%)$ \\
\hline \multirow{2}{*}{ Horas al día } & Menos de 1 & $12(7.4)$ \\
& Entre 1 y 2 & $30(18.4)$ \\
& Entre 2 y 3 & $34(20.9)$ \\
& Entre 3 y 5 & $35(21.5)$ \\
& Más de 5 & $36(22.1)$ \\
& Todo el día & $16(9.8)$ \\
\hline Franja horaria & De 8h a 14h. & $61(37.4)$ \\
& De 14h a 21h & $155(95.1)$ \\
& De 21h a 24h & $133(81.6)$ \\
\hline
\end{tabular}

Tabla 3

Resultados de las correlaciones bivariadas en la submuestra de hombres y mujeres

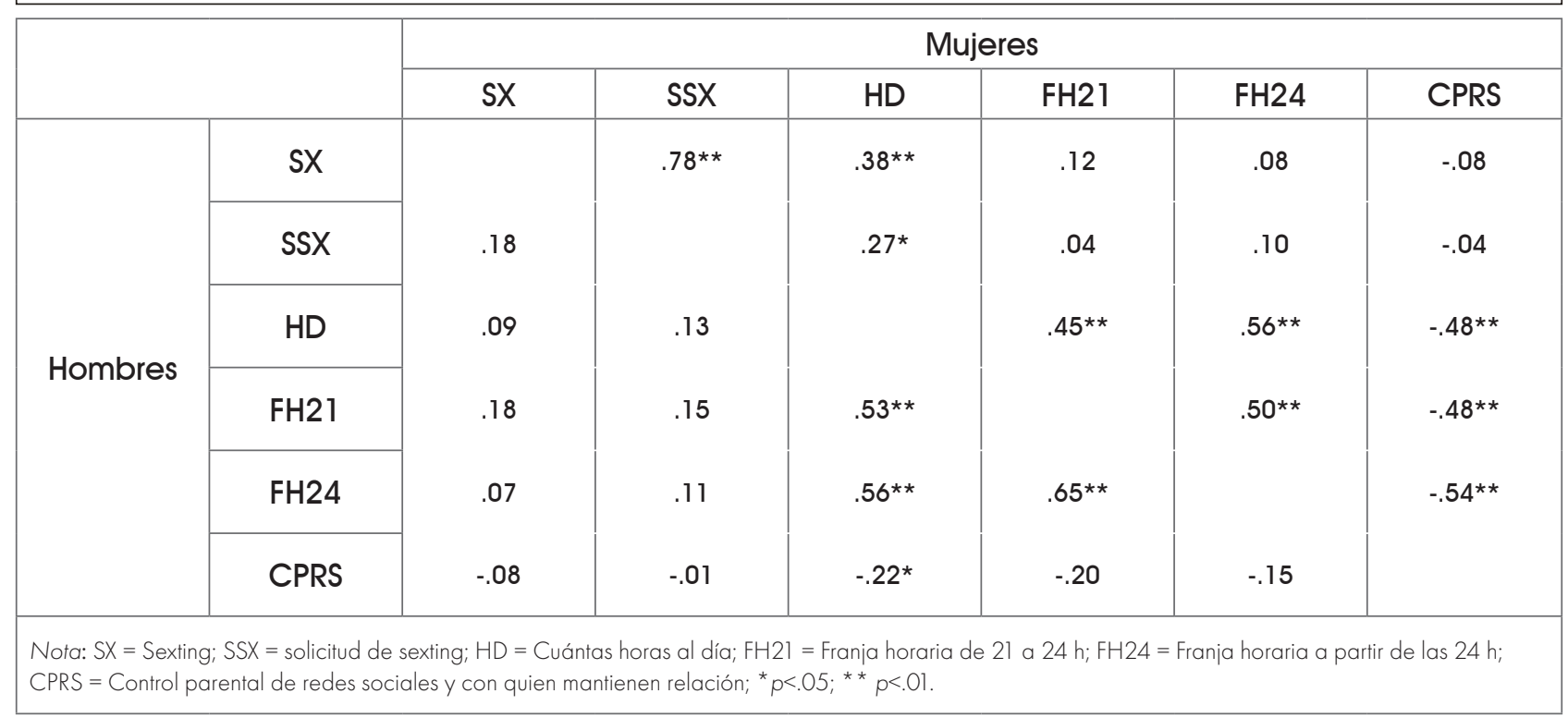

de redes sociales $(r=-.22 ; p=.03)$. Por su parte, en la submuestra de chicas, el sexting correlacionó positivamente con solicitud de sexting $(r=.78 ; p<.001)$ y con frecuencia de conexión a internet $(r=.38 ; p=.002)$. Solicitud de sexting correlacionó positivamente con frecuencia de conexión a internet $(r=.27$; $p=.03)$. La frecuencia de conexión a internet correlacionó positivamente con franja horaria de conexión de 21 a 24 horas $(r=.45 ; p<$ $.001)$ y a partir de las 24 horas $(r=.56 ; p<$ $.001)$; y también correlacionó negativamente con el control parental de redes sociales $(r=$ -.48; $p<.001)$. Franja horaria de conexión de 21 a 24 horas correlacionó positivamente con franja horaria de conexión a partir de las 
24 horas $(r=.50 ; p<.001)$ e igualmente, correlacionó negativamente con el control parental de redes sociales $(r=-.48 ; p<.001)$. Por último, franja horaria de conexión a partir de las 24 horas correlacionó negativamente con el control parental de redes sociales $(r=$ $-.54 ; p<.001)$.

En las pruebas multivariante del ANCOVA, los resultados observados (Tabla 4) mostraron significación para el modelo planteado, tanto en hombres $\left(F_{(2.95)}=44.09 ; p=.001 ; \eta^{2}=\right.$ $.50)$ como en mujeres $\left(F_{(2.68)}=5.49 ; p=.006\right.$; $\left.\eta^{2}=.15\right)$. Sin embargo, sólo en mujeres se observó significación para dos de las variables

\section{Tabla 4}

Resultados de las pruebas multivariante en el ANCOVA

\begin{tabular}{|l|l|c|c|c|}
\hline Género & \multicolumn{1}{|c|}{ VI } & $F$ & $\mathrm{P}$ & $\eta^{2}$ \\
\hline \multirow{5}{*}{ Hombre } & Interceptación & 44.08 & .00 & .50 \\
& HD & 0.16 & .85 & .00 \\
& FH24 & 0.90 & .41 & .02 \\
& CPRS & 1.70 & .19 & .04 \\
& FH24*CPRS & 2.14 & .12 & .05 \\
\hline \multirow{5}{*}{ Mujer } & Interceptación & 5.49 & .01 & .15 \\
& HD & 5.66 & .01 & .15 \\
& FH24 & 2.5 & .09 & .08 \\
& CPRS & 2.27 & .07 & .08 \\
& FH24*CPRS & 4.00 & .02 & .11 \\
\hline
\end{tabular}

Nota: HD = Cuántas horas al día; FH24 = Franja horaria a partir de las $24 \mathrm{~h} ; \mathrm{CPRS}=$ Control parental de redes sociales y con quien mantienen relación; $p<.05$.

independientes contempladas, siendo franja horaria de conexión a partir de las 24 horas ( $F$ $\left.{ }_{(2.68)}=5.66 ; p=.006 ; \eta^{2}=.15\right)$ y la interacción de esta variable con control parental de redes sociales $\left(F l_{2.68)}=4.00 ; p=.023 ; \eta^{2}=.11\right)$. A continuación se presentan los resultados hallados en las pruebas de efectos inter-sujetos del ANCOVA para las variables de estudio (Tabla 5 y Tabla 6).

En el caso de los hombres, el modelo no fue significativo, tanto para sexting como para solicitud de sexting. Sin embargo, en el caso de las mujeres, el modelo fue significativo tanto para sexting $\left(F_{(1.68)}=3.96 ; p=.006 ; \eta^{2}=\right.$ $.20)$, como para solicitud de sexting $\left(F_{(1.68)}=\right.$ $\left.3.48 ; p=.012 ; \eta^{2}=.18\right)$. Por su parte, con
Tabla 5

Resultados de los efectos inter-sujeto en el ANCOVA para la variable dependiente Sexting

\begin{tabular}{|l|l|c|c|c|}
\hline Género & \multicolumn{1}{|c|}{ VI } & $\mathrm{F}$ & $\mathrm{P}$ & $\eta^{2}$ \\
\hline \multirow{5}{*}{ Hombre } & Modelo & 0.61 & .66 & .03 \\
& corregido & & & \\
& Interceptación & 22.07 & .00 & .20 \\
& HD & 0.11 & .75 & .00 \\
& FH24 & 0.61 & .44 & .01 \\
& CPRS & 1.60 & .21 & .02 \\
& FH24*CPRS & 1.30 & .26 & .01 \\
\hline \multirow{5}{*}{ Mujer } & Modelo & 3.96 & .01 & .20 \\
& corregido & 9.21 & .00 & .13 \\
& Interceptación & 90 & .15 \\
& HD & 10.73 & .00 & .05 \\
& FH24 & 3.60 & .06 & .05 \\
& CPRS & 1.52 & .22 & .02 \\
& FH24*CPRS & 2.46 & .12 & .04 \\
\hline
\end{tabular}

Nota: $\mathrm{HD}=$ Cuántas horas al día; FH24 = Franja horaria a partir de las $24 \mathrm{~h} ; \mathrm{CPRS}=$ Control parental de redes sociales $y$ con quien mantienen relación; $p<.05$.

\section{Tabla 6}

Resultados de los efectos inter-sujeto en el ANCOVA para la variable dependiente Solicitud de sexting

\begin{tabular}{|c|c|c|c|c|}
\hline Género & VI & $F$ & $p$ & $\eta^{2}$ \\
\hline \multirow{6}{*}{ Hombre } & $\begin{array}{l}\text { Modelo } \\
\text { corregido }\end{array}$ & 1.37 & .25 & .06 \\
\hline & Interceptación & 77.39 & .00 & .46 \\
\hline & HD & 0.27 & .61 & .00 \\
\hline & FH24 & 1.45 & .23 & .02 \\
\hline & CPRS & 2.35 & .13 & .03 \\
\hline & $\mathrm{FH} 24{ }^{\star} \mathrm{CPRS}$ & 3.57 & .06 & .04 \\
\hline \multirow{6}{*}{ Mujer } & $\begin{array}{l}\text { Modelo } \\
\text { corregido }\end{array}$ & 3.48 & .01 & .18 \\
\hline & Interceptación & 10.29 & .00 & .13 \\
\hline & $\mathrm{HD}$ & 3.63 & .06 & .06 \\
\hline & $\mathrm{FH} 24$ & 5.08 & .03 & .08 \\
\hline & CPRS & 5.07 & .03 & .07 \\
\hline & $\mathrm{FH} 24^{*} \mathrm{CPRS}$ & 7.52 & .01 & .11 \\
\hline \multicolumn{5}{|c|}{$\begin{array}{l}\text { Nota: } \mathrm{HD}=\text { Cuántas horas al día; } \mathrm{FH} 24=\text { Franja horaria a partir de las } \\
24 \text { h; CPRS = Control parental de redes sociales y con quien mantienen } \\
\text { relación; } p<.05 \text {. }\end{array}$} \\
\hline
\end{tabular}

relación a sexting, se halló significación en la variable predictora frecuencia de conexión ( $F$ $\left.{ }_{(1.68)}=10.73 ; p=.002 ; \eta^{2}=.15\right)$. En relación a la solicitud de sexting, se halló significación en las variables predictoras franja horaria de 
conexión a partir de las 24 horas $\left(F_{(1.68)}=5.08\right.$; $\left.p=.028 ; \eta^{2}=.08\right)$, control parental de redes sociales $\left(F_{(1.68)}=5.07 ; p=.028 ; \eta^{2}=.07\right)$, así como la interacción entre ambas $\left(F_{(1.68)}=7.53\right.$; $\left.p=.008 ; \eta^{2}=.11\right)$.

\section{DISCUSIÓN}

En el presente estudio, se ha encontrado que en torno al $32 \%$ de los adolescentes muestran una frecuencia de conexión a internet elevada (más de cinco horas al día); y un porcentaje igualmente destacable lo hace en horas nocturnas (el $81.6 \%$ entre las 21 y 24 horas, y el $40 \%$ a partir de las 24 horas). También, que cuanto mayor es la frecuencia de conexión, mayor es la probabilidad de estar conectado en horarios nocturnos (Tabla 3), observándose diferencias de género a este respecto en la franja horaria de 21 a 24 horas, siendo mayor ésta relación en el caso de los chicos que de las chicas. Este hallazgo puede explicarse por una mayor permisividad en el control parental ejercido sobre los chicos, de acuerdo a lo observado por Arnaiz et al., (2016).

En consonancia con lo expresado por Garmendia et al., (2016), los patrones de conexión de alta frecuencia a internet y en horario nocturno hallados en nuestro estudio aumentarían la probabilidad de exposición a riesgos de internet, como el sexting o la sextorsión. Además, estos pueden llevar a otros como el grooming y el ciberbullying (Fajardo, Gordillo y Regalado, 2013).

En nuestro estudio el $11 \%$ de los adolescentes han experimentado situaciones de sexting en los últimos 30 días en relación a haber recibido contenidos de tipo sexual, un $9.2 \%$ ha recibido solicitudes de compartir material sexual íntimo, mientras que el $0,6 \%$ ha experimentado sextorsión. Las prevalencias halladas son menores que las encontradas por Garmendia et al. (2016), que señalaban que un $31 \%$ de los jóvenes ha recibido mensajes con contenido sexual. Esta diferencia se explica porque en el estudio de Garmendia et al. (2016) los datos están referidos a los últimos 12 meses, mientras que en nuestro estudio hemos utilizado el tramo temporal de últimos 30 días, y porque en el citado estudio se incluye además mensajes escritos de contenido sexual dentro de la conceptualización de sexting. Otros estudios obtienen resultados en línea con nuestros hallazgos. Gutiérrez (2014) indica que el 15\% de los menores entre 12 y 17 años han sufrido este tipo de riesgos en internet a través de sus dispositivos. En Pérez et al. (2011) se informa que entre un 4 y $8 \%$ de los adolescentes entre 10 y 16 años señalaron haber experimentado sexting, bien como emisores de contenidos sexuales o como receptores de este.

En cuanto a las diferencias de género halladas en nuestro estudio, se observa que la prevalencia de sexting, solicitud de sexting y sextorsión es mayor en chicas que en chicos (Tabla 1). Este hecho puede explicarse a través de otros hallazgos observados, donde la frecuencia de conexión a internet mantiene una relación positiva con sexting y solicitud de sexting en el caso de las chicas, mientras que esta relación no se observa en chicos. De igual modo, en las pruebas multivariante del análisis ANCOVA se observa que la frecuencia de conexión a internet predice el sexting y solicitud de sexting en chicas, pero no en chicos; y que en las pruebas inter-sujetos predice igualmente el sexting en chicas. Estos datos indican que en aquellas chicas que muestran un patrón de mayor frecuencia de conexión aumenta la probabilidad de experimentar estos riesgos, respecto de sus iguales chicos con el mismo patrón de conexión.

Estos hallazgos están en consonancia con los de Chang et al. (2015) y Fernández-Montalvo et al. (2015) que señalan que las chicas utilizan más las redes sociales que los chicos, siendo a través de éstas donde se formulan las solicitudes de sexting y se realiza este. Aunque el sexting suele circunscribirse dentro de una relación de pareja, también puede producirse en el proceso de cortejo, tanto con personas conocidas como con otras recientemente contactadas a través de las redes sociales. En este sentido, Garmendia et al. (2016) informan que las chicas son más propensas a aceptar todo tipo de peticiones de amistad, y aunque generalmente acepten a 
otras personas conocidas, puede aumentar su vulnerabilidad si no se acompaña de estrategias autoregulatorias que eviten compartir archivos de carácter íntimo y sexual. Precisamente, puede ser la confianza en ser personas conocidas lo que disminuya la percepción de riesgo para compartir este tipo de contenidos íntimos.

Siendo así, es esencial desarrollar intervenciones educativas y preventivas que permitan a los adolescentes autorregularse ante el uso de internet, teniendo en cuenta la relevancia de la frecuencia de conexión a internet, a tenor de lo hallado en nuestro estudio. En relación a ello, como señalan Leung y Lee (2011), el control parental es un factor de protección sobre los riesgos de internet, sobre todo aplicado en relación al tiempo de conexión.

Atendiendo al control parental de redes sociales, en nuestro estudio se observan diferencias significativas con respecto a la frecuencia de horas de conexión a internet, y en las franjas horarias de conexión entre 21:00 y 24:00 horas, y a partir de las 24:00 horas. Mientras que en chicas se observa una relación significativa inversa para las tres variables citadas, en el caso de los chicos sólo se observa esta relación con la frecuencia de horas de conexión a internet. Es decir, las chicas que refieren un mayor control parental de redes sociales, muestran una menor frecuencia de conexión a internet y menor tasa de conexión en horarios nocturnos. Sin embargo, en chicos este control es menor, limitándose a la frecuencia de horas de conexión, y no a los horarios. Este hallazgo está en consonancia con lo observado por Arnaiz et al. (2016) y Garmendia et al. (2016), los cuáles informaban que las chicas tenían mayor control parental que los chicos. En consecuencia, el control parental de redes sociales es un factor protector frente a la alta frecuencia de horas de conexión, tanto en chicos como en chicas, pero también lo es en relación a la frecuencia de conexión en franjas horarias nocturnas en chicas, no así en chicos.

Asimismo, este hallazgo resulta especialmente relevante, si consideramos que es en estos horarios nocturnos cuando existe una mayor probabilidad de realizar acciones de sexting en el contexto de la intimidad de la habitación propia individual y un menor grado de interacción familiar.

Por otra parte, el control parental de redes sociales no se ha mostrado predictor del sexting ni en chicos ni en chicas, aunque sí para la solicitud de sexting en chicas. Es decir, el control parental se muestra como un factor protector frente a la solicitud de sexting únicamente en chicas. Sin embargo, cuando se ha considerado la interacción del control parental de redes sociales junto con la franja horaria de conexión a partir de las 24 horas, dicha interacción se ha mostrado significativa tanto en las pruebas multivariante del ANCOVA, como para la solicitud de sexting, de nuevo únicamente en chicas.

Como ya se ha indicado anteriormente, el control parental de redes sociales se relaciona con una menor frecuencia de conexión a internet en la franja horaria a partir de las 24 horas en chicas, no siendo así en chicos. Por tanto, se concluye que en el caso de las chicas, la medida restrictiva de horarios nocturnos de conexión a internet aplicada a través del control parental es una medida eficaz para reducir la probabilidad de exposición a solicitudes de sexting; y, en consecuencia, a compartir imágenes íntimas con las que posteriormente poder ser víctima de ciberbullying o sextorsión.

A pesar de ello, el presente estudio muestra la existencia de diferencias de género en relación a la prevalencia de experimentar sexting, solicitud de sexting y sextorsión, estando más expuestas las chicas que los chicos. Esto sugiere la existencia de otros factores intervinientes no contemplados en este estudio, así como la posibilidad de que no se ejerza habitualmente o adecuadamente el control parental de redes sociales en chicas. Esto refuerza la idea de que es necesario impulsar intervenciones educativas orientadas al ámbito familiar, y reforzadas en el ámbito escolar, que desarrollen el conocimiento y entrenamiento en medidas de control parental, y en su aplicación eficaz.

A modo de conclusión, considerando que hay otra serie de factores causales que podrían explicar 
la relación entre patrones de uso de internet, control parental y sexting, lo hallado en el presente estudio apunta a que una mayor frecuencia de conexión a internet es un factor de riesgo para sexting y sextorsión, pero únicamente para mujeres. Por su parte, el control parental de redes sociales se relaciona con una menor frecuencia de conexión a internet, tanto en chicos como en chicas. Sin embargo, cuando se combinan control parental de redes sociales con medidas restrictivas de frecuencia de horas de conexión a internet y franja horaria nocturna de conexión, disminuye la probabilidad de exposición al sexting y la sextorsión, pero únicamente en chicas.

Tal como señaló Mesch (2009), aquellos adolescentes que tenían mayor control parental, mostraban menos conductas de riesgo, al disponer de reglas y normas adecuadas sobre el uso. Por ello, es importante impulsar estrategias educativas y preventivas a nivel familiar y escolar que fomenten un uso adecuado de internet, el conocimiento de las posibilidades y riesgos, así como el establecimiento de estrategias de mediación y control parental que promuevan el empoderamiento y autorregulación de los adolescentes frente a los riesgos de internet.

Finalmente, son varias las limitaciones que presenta este estudio. Por una parte, el tipo de asignación de los participantes a los grupos, donde lo ideal es que fuese al azar, y con un tamaño de muestra mayor, así como el uso de instrumentos no validados previamente, si bien no se miden constructos sino conductas concretas. Asimismo, si bien nuestro estudio permite destacar la relevancia de la perspectiva de género en el estudio del sexting y de los factores de riesgo para este y otros riesgos de internet y redes sociales, es preciso realizar estudios que amplíen el conocimiento en esta línea, así como otros de carácter longitudinal que permitan establecer su influencia a lo largo de la trayectoria vital de los adolescentes. Esto permitirá su aplicación en el ámbito educativo y preventivo, ajustando las intervenciones en función del género, haciéndolas más efectivas.

\section{- Conflicto de intereses}

Los autores declaran no tener ningún conflicto de intereses.

\section{REFERENCIAS}

Arnaiz, P., Cerezo, F., Giménez, A.M. y Maquilónz, J.J. (2016). Conductas de ciberadicción y experiencias de cyberbullying entre adolescentes. Anales de psicología, 32(3), 761-769. doi: http://dx.doi. org/10.6018/analesps.32.3.217461

Arab, E. y Díaz, A. (2015). Impacto de las redes sociales e internet en la adolescencia. Aspectos positivos y negativos. Revista Médica Clínica Las Condes, 26 (1), 7-13.

Chang, F.C., Chiu, C.H., Miao, N.F., Chen, P.H., Lee, C.M., Chiang, J.T. y Pan, Y.C. (2015). The relationship between parental mediation and Internet addiction among adolescents, and the association with cyberbullying and depression. Comprehensive Psychiatry, 57, 21-28. doi: https://doi.org/10.1016/i. comppsych.2014.11.013

De la Villa Moral, M. y Suárez, C. (2016). Factores de riesgo en el uso problemático de internet y del teléfono móvil en adolescentes españoles. Revista Iberoamericana de Psicología y Salud, 7, 69-78. doi: http:// dx.doi.org/10.1016/i.rips.2016.03.001

Ding, Q., Li, D., Zhou, Y., Dong, H. y Luo, J. (2017). Perceived parental monitoring and adolescent Internet addiction: A moderated mediation model. Addictive Behaviors, 74, 48-54. doi: http://dx.doi.org/10.1016/i. addbeh.2017.05.033

Duerager, A., y Livingstone, S. (2012). How can parents support children' s internet safety? EU Kids online. London, UK. Recuperado de http://eprints.Ise.ac.uk/42872/

Echeburúa, E. (2013, mayo-junio). Atrapados en las redes sociales. Critica. Recuperado de http://www.revista-critica.com/la-revista/ monografico/analisis/46-atrapados-enlas-redes-sociales

Fajardo, I., Gordillo, M. y Regalado, A.B. (2013). Sexting: Nuevos usos de la tecnología y la sexualidad en adolescentes. International Journal of Developmental anda Educational Psychology. Familia y educación: aspectos positivos, 1 (1), 521 533. 
Fernández-Montalvo, J., Peñalva, A. e Irazabal, I. (2015). Hábitos y conductas de riesgo en internet en la preadolescencia. Comunicar, 22 (44), 113-120. doi: http://dx.doi. org/10.3916/C44-2015-12

Garmendia, M. Jiménez, E., Casado, M.A. y Mascheroni, G. (2016). Net Children Go Mobile: Riesgos y oportunidades en internet y el uso de dispositivos móviles entre menores españoles (2010-2015). Madrid: Red.es/ Universidad del País Vasco/Euskal Herriko Unibertsitatea

Gómez, P., Rial, A., Braña, T., Golpe, S. y Varela, J. (2017). Screening of Problematic Internet Use among Spanish adolescents: Prevalence and related variables. CyberPsychology, Behavior, and Social Networking, 20(4), 259-267. doi: 10.1089/cyber.2016.0262

Gutiérrez I.M. (2014). Cyberbullying y sexting: Percepción y propuestas de estudiantes universitarios. Multidisciplina, (17), 93-119.

Khurana, A., Bleakley, A., Jordan, A.B. y Romer, D. (2015). The Protective Effects of Parental Monitoring and Internet Restriction on Adolescents'Risk of Online Harasment. Journal Youth Adolescence, 44(15), 1039 1047. doi: 10.1007/s10964-014-0242-4 Lau, W.W. y Yuen, A.H. (2016). The relative importance of paternal and maternal parenting as predictors of adolescents 'home Internet use and usage. Computers \& Education, 102, 224-233. doi: http://dx.doi. org/10.1016/i.compedu.2016.09.002
Leung, L. y Lee, P.S. (2011). The influences of information literacy, Internet addiction and Parenting Styles on Internet risks. New Media \& Society, 1-21. doi: $10.1177 / 1461444811410406$

Mascheroni, G., Murru, M.F., Aristodemou, E., \& Laouris, Y. (2013). Parents. Mediation, self-regulation and co-regulation. In B. O’Neill, E. Staksrud, \& S. McLaughlin (eds.) Towards a better internet for children? Policy pillars, players and paradoxes (pp.211225). Göteborg: Nordicom.

Mesch, G.S. (2009). Parental Mediation, Online Activities, and Ciberbullying. CyberPsychology \& Behavior, 12(4), 387 393. doi: $10.1089 / \mathrm{cpb} .2009 .0068$

Moodley, P. y Singh, R.J. (2016). Parental regulation of Internet use: Issues of control, censorship and cyberbullying. Mousaion, 34(2), 15-30.

Pérez, P., Flores, J., de la Fuente, S., Álvarez, E., García, L., y Gutiérrez, C. (2011) Guía sobre adolescencia y sexting: qué es y cómo prevenirlo. Observatorio de la Seguridad de la Información de INTECO y PantallasAmigas.

Sureda, J., Comas, R. y Morey, M. (2010). Menores y acceso a Internet en el hogar: las normas familiares. Comunicar, 17(34), 135-143. doi: 10.3916/C34-2010-03-13 